A. Pramesh Rao, G. Swarup and Gopal-Krishna, eds.

\title{
Spectral Mapping of Classical Double Radio Sources
}

\author{
J.P. Leahy and T.W.B. Muxlow \\ University of Manchester, Jodrell Bank Observatory, Macclesfield, \\ Cheshire, SK11 9DL, England
}

\begin{abstract}
We review the evidence that spectral curvature in the extended emission of radio galaxies is caused by synchrotron losses, and that the spatial variation can be interpreted to yield ages and expansion speeds. One of the biggest worries has been the true value of the magnetic field, but X-ray detections of inverse-Compton radiation are beginning to suggest that "minimum energy" estimates are remarkably accurate. A critical test is to compare model and observed spectra over a broad frequency range; to date this has has only been done for Cygnus A, and the results proved controversial. Here we discuss several more cases and begin to draw some general conclusions.

Hotspots are usually well fitted by continuous injection models, as expected. In two cases the implied injection index is flatter than 0.5 , too flat to be produced by standard Fermi acceleration in a non-relativistic shock. The bridge spectra are reasonably fitted by single-burst models, but in some objects the injection index is not constant across the lobes, showing instead a tendency to steepen in the inner bridge, where the break frequencies are lowest. The true spectral shape may be a more gradual curve than the standard models, possibly because of mixing of electron populations with different ages. Our results are limited by the inaccuracy of the absolute flux density scale, especially at low frequencies, and a fresh attack on the flux scale would be timely.
\end{abstract}

\section{Introduction}

Classical double radio sources show a characteristic steepening of the two-point spectral index from the hotspots back towards the nucleus (e.g. Leahy, Muxlow \& Stephens 1989; Dennett-Thorpe et al. 1997), as shown in Fig. 1. Multifrequency studies, starting with Burch (1977), show that in fact the spectra are curved, so the variation of spectral index is better interpreted as the variation of a "break frequency", $\nu_{T}$, at which the spectral steepening sets in. Since this effect was discovered it has been interpreted in terms of synchrotron ageing of the spectrum (Kardashev 1962), but the results of such analyses remain controversial (Rudnick 2000). Given these uncertainties it is important to check the theory via detailed comparisons with real data. Here, after a brief review of the theory, we do just that, and discuss some of the implications. Finally, we argue that one of the most worrying uncertainties in age estimates, namely the value of the magnetic field, may be on surprisingly firm ground. 


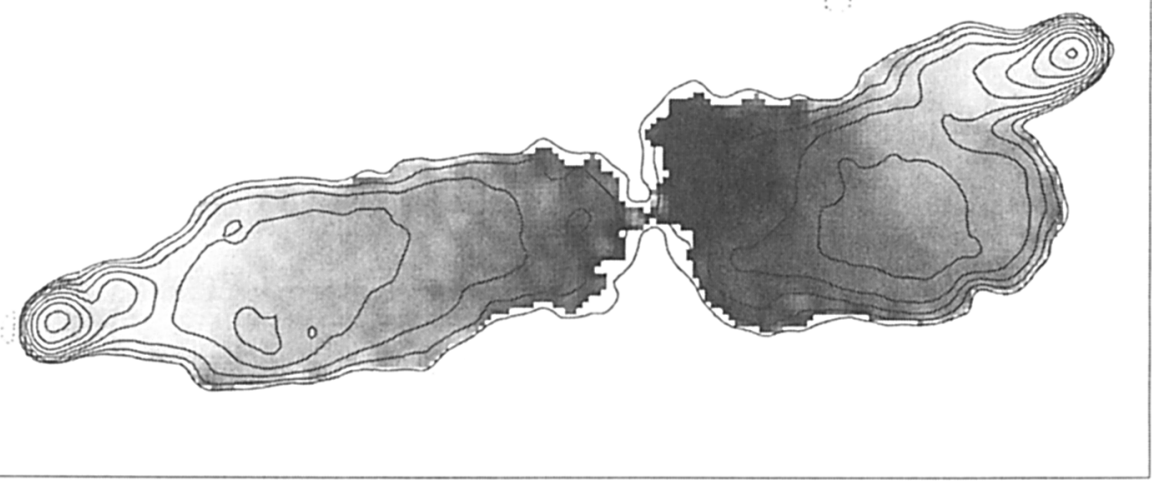

Figure 1. Spectral index $\alpha$ (where $S_{\nu} \propto \nu^{-\alpha}$ ) of 3C 79 between 1.4 and $8.4 \mathrm{GHz}$. The greyscale runs from $\alpha=0.65$ (white) to 1.8 (black), and is plotted where the error is less than 0.2 . The radio core has been subtracted. Contours are of total intensity at $1.4 \mathrm{GHz}$.

\section{Theory of Spectral Ageing}

The simplest models of aged synchrotron spectra start from an initial power law spectrum, and differ mainly according to whether the power law is injected continuously (CI models) or instantaneously ("one-shot" models). Leahy (1991) gives more details and references. The spectrum evolves self-similarly with $\nu_{T}$ shifting to lower frequency over time. In a constant magnetic field, $B$, the time since injection is

$$
t=50 \mathrm{Myr} \times \frac{\sqrt{B}}{B^{2}+B_{\text {phot }}^{2}} \frac{1}{\sqrt{\nu_{T}}},
$$

where $\nu_{T}$ is in $\mathrm{GHz}, B$ in $\mathrm{nT}$, and $B_{\text {phot }}$ is the equivalent magnetic field (in $\mathrm{nT}$ ) to the photon energy density, present because of inverse-Compton losses. Note that $t$ has a maximum value (for $B=B_{\text {phot }} / \sqrt{3}$ ), which is surprisingly short ( $\sim 20 \mathrm{Myr}$ ) for sources observed at $z \sim 1$ with $\nu_{T} \sim 1 \mathrm{GHz}$ (Leahy et al. 1989).

Rudnick (2000) has emphasised the variety of ways that electrons can gain or lose energy in a lobe, arguing that this puts the whole concept of spectral ageing in doubt. But given the short maximum lifetimes and the timescales inferred, for instance, from ram-pressure arguments, radiative losses certainly ought to have a notable effect on the spectrum. It therefore seems perverse to doubt that spectral curvature is principally due to ageing: one would need to invoke an unknown particle acceleration process which has an identical energydependence to synchrotron losses to prevent spectral ageing. Rather than give up entirely, let us see what effect relaxing the assumptions of the model has on the resulting ages. 


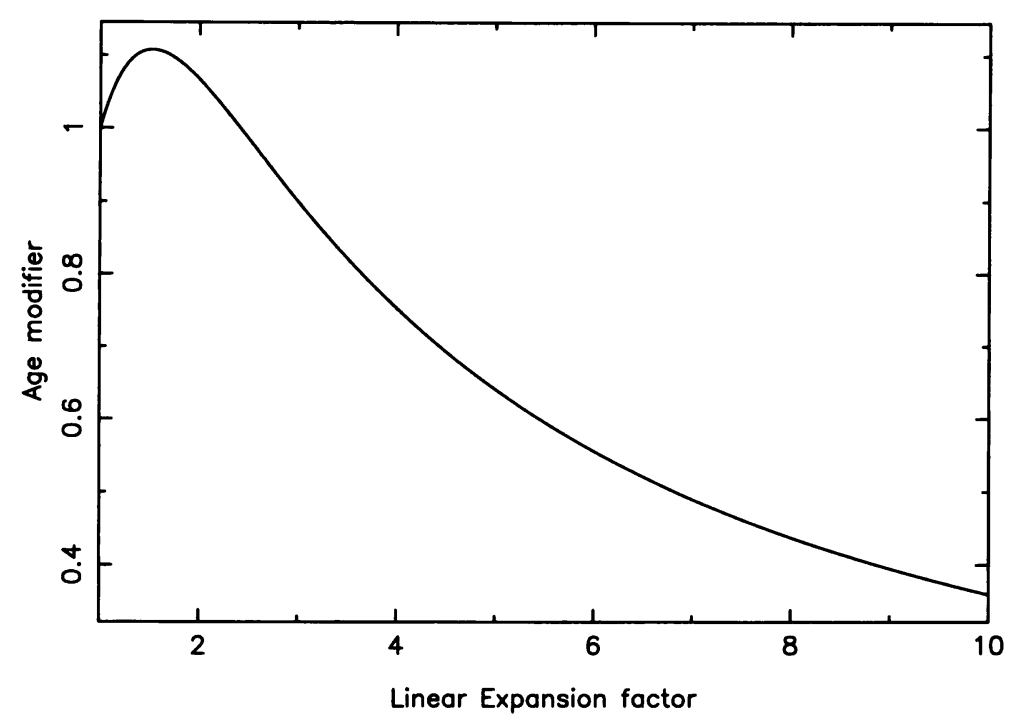

Figure 2. Effect of steady adiabatic expansion on derived spectral age: Curve gives the ratio of derived to true age.

Clearly, it is an idealisation to assume a constant magnetic field. In numerical models the plasma is maximumally compressed by the jet terminal shock (identified with the hotspot, often assumed to be the site of particle acceleration), and expands thereafter. The case of steady expansion and simultaneous ageing was considered by Kardashev (1962) and Alexander (1987). Two effects compete: expansion weakens the magnetic field and hence reduces the rate of ageing, but also the radiating electrons suffer adiabatic losses, which lowers the break frequency. Fig. 2 shows the ratio of the derived to the true age, if one assumes a constant $B$ equal to the initial value, and effective pitch-angle scattering. As pointed out by Alexander (1987), for expansion by less than a factor of 4 (i.e. 64 in the volume), the derived age is good to within $25 \%$. Larger expansion factors would in practice make the source invisible.

Somewhat larger effects can occur if one realistically allows particles to travel between regions of different field strengths. This case was treated by Tribble (1993). Here the form of the spectrum is modified, but the major changes to the spectrum occur at frequencies above $\nu_{T}$, where the general effect is to reduce the steepening. A mix of ages along the line of sight or within the telescope beam has the same effect (Tribble 1993). The reason is obvious: such models can be considered as superpositions of the basic aged spectrum with different $\nu_{T}$, and because this falls steeply at high frequency, the summed spectrum at high frequency will be dominated by the "youngest" components, while at low frequency all components will enter with equal weight. The crucial question here is how far wrong the derived break frequency would be, if conventional spectral models are fitted to these modified spectra. A point to bear in mind is that at frequencies more than a few times $\nu_{T}$, all one-shot models predict a flux so low that it cannot be measured accurately, so the observational constraints 
refer mainly to frequencies up to around $\nu_{T}$. We have not yet investigated this issue numerically, but examination of the plots given by Tribble suggest that the break frequency would generally be in error (usually too high) by only a factor of $<4$, corresponding to an underestimate in age of less than a factor of two. This refers to a weighted average age if there is a mixture; this will differ from the more interesting oldest age by another factor of less than two (a factor of two would correspond to continuous injection, in which case the spectrum is different enough that it can be clearly distinguished, as we shall see). In summary derived ages are likely to be underestimates, but by less than a factor of three.

These considerations also mean that the traditional question of whether or not the spectra show evidence of pitch-angle scattering ("Jaffe \& Perola" vs. "Kardashev-Pacholczyk" models, hereafter JP and KP) is something of a red herring, because this distinction (again, and for the same reason) only shows up clearly above $\nu_{T}$, where the spectra are likely to be somewhat flattened in any case by the complications discussed by Tribble.

\section{Observations}

The predicted spectral curvature is very gradual, except at the high frequency end where the intensity plummets with consequent loss of accuracy. With typical synthesis images, one needs observations covering about a decade of frequency even to break the degeneracy between the injection spectral index $\alpha_{0}$ and break frequency, so in most analyses it has simply been assumed that $\alpha_{0}$ is constant across the radio structure. Some evidence that this might be the case comes from the low-frequency integrated spectra of radio sources (e.g. Laing \& Peacock 1980); variation in $\alpha_{0}$ would inevitably lead to a concave ('u'-sense) spectrum at low frequencies, but in general the spectra are straight or convex (' $n$ '-sense).

To go further and test whether the spectrum is actually of the assumed form requires even broader frequency coverage. We have observed eight objects selected from "3CRR" (Laing, Riley \& Longair 1983) over two spectral decades, chosen because they showed a simple classical double structure, $S_{178 \mathrm{MHz}}>19 \mathrm{Jy}$, and a size $\sim 1^{\prime}$, allowing us to map them with roughly matched resolution with MERLIN at $151 \mathrm{MHz}$ and $408 \mathrm{MHz}$, and the VLA at all bands from 0.3 to $15 \mathrm{GHz}$. In practice the largest scale is undersampled at $408 \mathrm{MHz}$ while the resolution is significantly worse at $330 \mathrm{MHz}$. This range of frequency coverage has only previously been matched in the single case of Cygnus A (Carilli et al. 1991). Analysis of this database is still in progress, but here we summarise some of the initial results.

\subsection{Flux Density Scale}

With moderate care, but no elaborate observational procedures, one can transfer flux from VLA calibrators to the targets with better than $0.5 \%$ repeatability. This is much better than the estimated errors on the absolute flux density scale, which are $2 \%$ between 0.3 and $30 \mathrm{GHz}$, and $5 \%$ at lower frequencies (Baars et al. 1977). Initially, we assigned a minimum error to each image on the basis of the Baars et al. uncertainty, but we found that $\chi_{r}^{2} \ll 1$ at most hotspots. In the following plots we have therefore assigned a $1 \%$ scale uncertainty between 1.4 and $15 \mathrm{GHz}$, which still tends to give too-low values for $\chi_{r}^{2}$. 

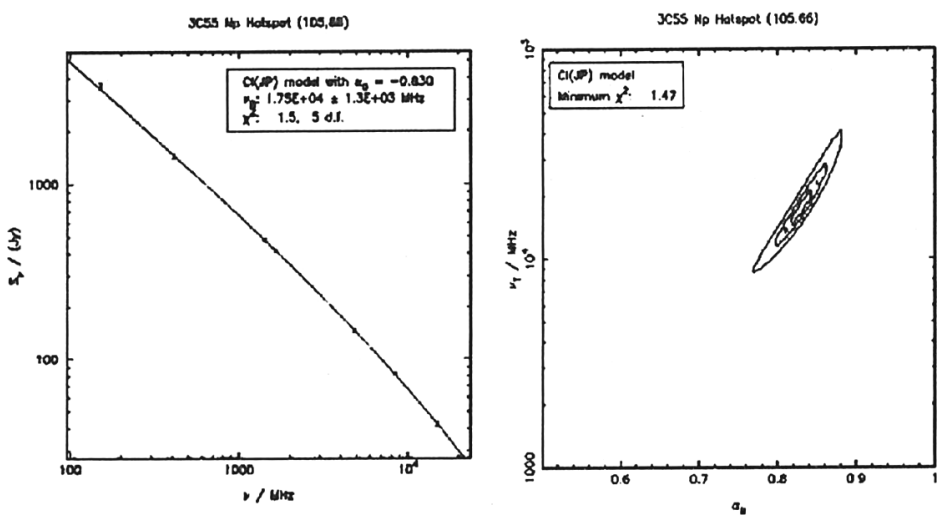

Figure 3. Left: Spectrum of the Sf secondary hotspot of 3C 55, with the best-fit continuous injection model superposed. Right: Contours of $\chi^{2}$ for the model fit in the $\alpha_{0}, \nu_{T}$ plane. Contours are at $1,2.71$ and 6.63 above the minimum $\chi^{2}$ value (i.e. the formal 68,90 and $99 \%$ confidence contours for 1 parameter).

This does not mean that the Baars et al. scale is much more accurate than claimed; rather, by fitting log-parabolic spectra to the raw data they ensure that the errors are strongly correlated in frequency: an overall scale error would not affect our results, but there will also be a slight spectral index error caused by deviations of the true spectra of the primary calibrators from the assumed functional form.

The upshot of all this is that for bright objects the uncertainty of the flux scale is the dominant source of error in extracting astrophysical information from radio spectra, especially at low frequencies. We hope that the important results from low-frequency work presented at this symposium will inspire a fresh attack on the flux scale: the present one is based on 1960s-vintage measurements, and we can now do much better in two areas. At decimetre to metre wavelengths, the improvement in receiver sensitivity should allow more accurate measurements with simple antennas suitable for absolute measurements; while the improvement in computing power means that we ought to be able to use rather larger and more complex antennas both here and, crucially, at lower frequencies.

\section{Results: Hotspots}

The hotspot spectra are the easiest to determine because they are well sampled at all frequencies, and of course are the brightest features. Most hotspots are fitted to remarkable accuracy by continuous injection models, showing the characteristic break of 0.5 in spectral index (e.g. Fig 3 ). In two cases, the injection index fitted was clearly flatter than 0.5 , which is the limiting case for conventional non-relativistic Fermi acceleration (e.g. Heavens 1989). The most 
3C268.1 Np Hotspot $(88,65) \mathrm{C}$

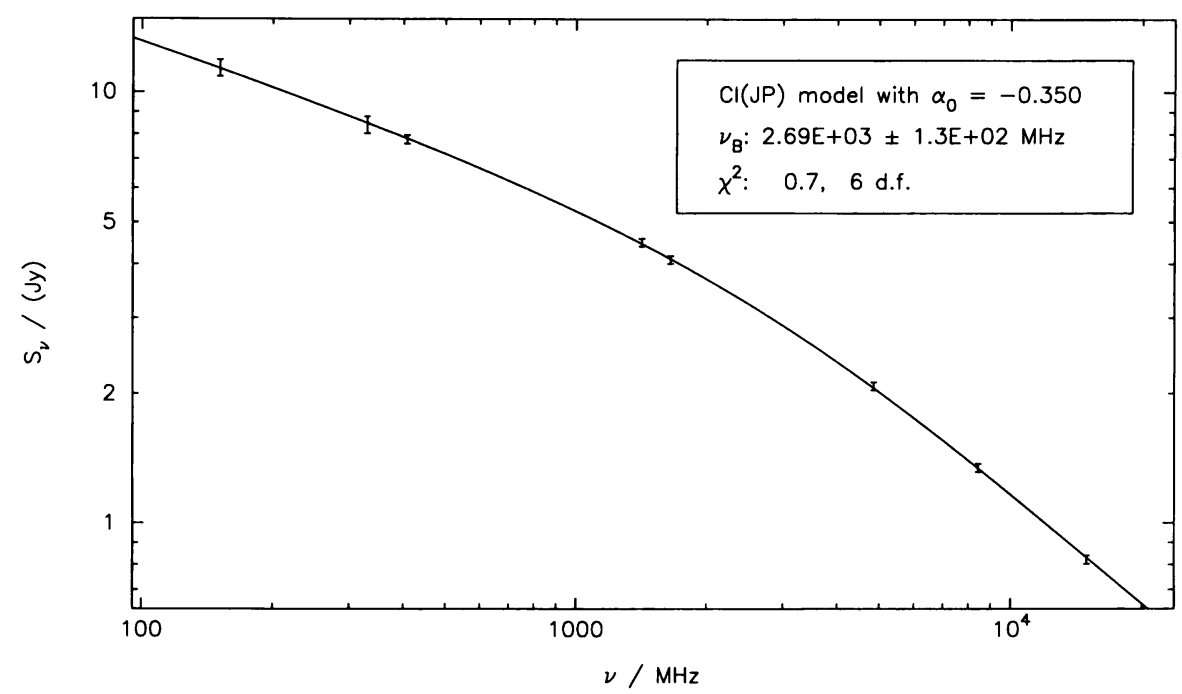

Figure 4. Spectrum of the Np hotspot of 3C 268.1; details as for Fig. 3.

extreme of these is 3C 268.1 (Fig. 4), with $\alpha_{0}=0.35 \pm 0.03$. Also, 3C $79 \mathrm{Sf}$ has a nearly straight spectrum which could be fit with $\alpha_{0}=0.43$ and $\nu_{T} \approx 270 \mathrm{MHz}$, but a solution with $\alpha_{0}=0.87$ and very high $\nu_{T}$ seems more plausible. Several options for producing such flat spectra are now available (Heavens 1989, Kirk \& Heavens 1989).

The $\chi^{2}$ contours plotted in Fig. $3 \mathrm{~b}$ show the residual observational correlation between the fitted $\alpha_{0}$ and $\nu_{T}$; however with the spectral range available for our data both parameters are relatively well determined, at least in the context of this model. There also seems to be a real correlation between these two quantities: Fig. 5 shows our results for the six sources where analysis is complete. The bracketed points (for 3C 330) have high $\chi^{2}$, probably due to a calibration error at $15 \mathrm{GHz}$; but exclusion of this frequency does not greatly affect the best fit. We can think of no selection effect that would discriminate against very flatspectrum hotspots with high break frequencies. One can invent models with continuous injection which show a correlation between $\nu_{T}$ and $\alpha_{0}$; for instance hotspots with strong relativistic shocks are expected to give the flattest spectra due to Fermi acceleration (Heavens 1989), and these might well have the highest post-shock magnetic fields and hence the most rapid ageing of the material as it flows through the hotspots. It is more difficult to come up with realistic tests of such models.

The majority of hotspots have break frequencies high enough that the lowfrequency limit is a convincing power-law (e.g. Fig 3); therefore the spread in $\alpha_{0}$ rules out the possibility that all are fitted by some universal slowly-curved spectrum, of the sort discussed by Katz-Stone, Rudnick \& Anderson (1993). The high-frequency limit is less-well constrained in most of our data. 


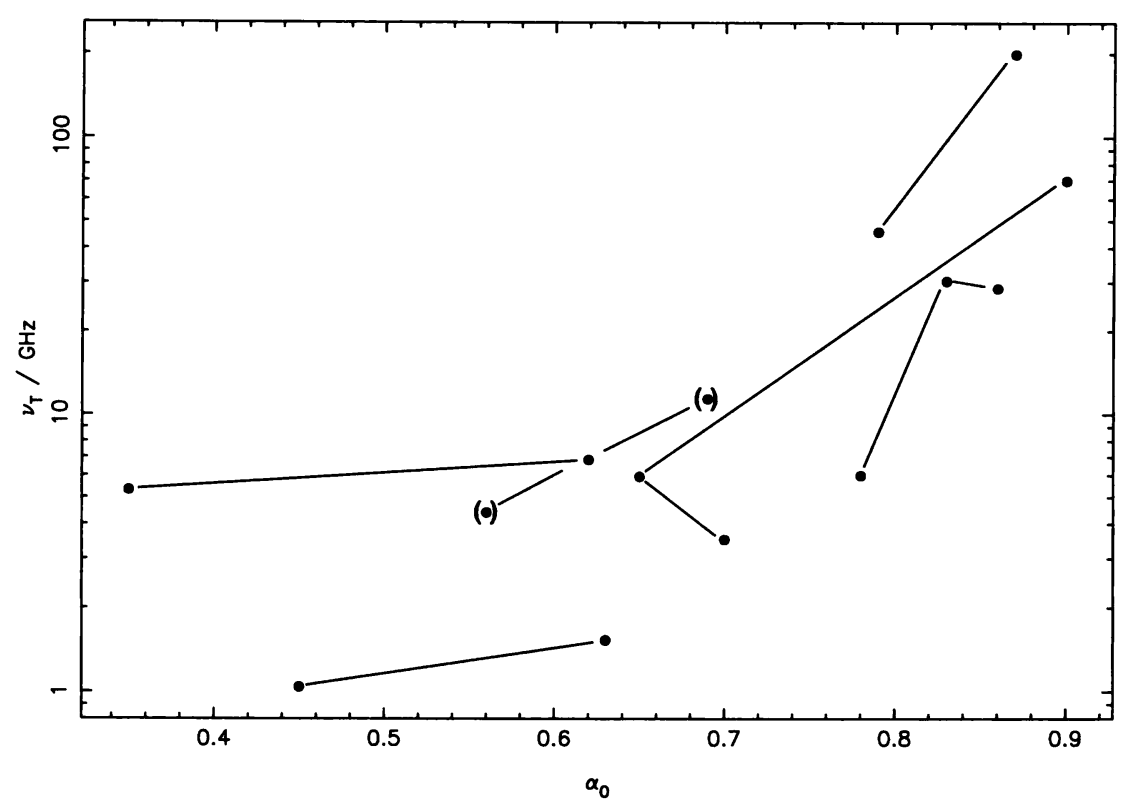

Figure 5. Plot of break frequency $\left(\nu_{T}\right)$, in the emitting frame, vs. injection spectral index $\left(\alpha_{0}\right)$. Lines join the hotspots for each source (two sources have double hotspots at one end).

\section{Results: Bridges}

All the bridges we have examined show greater spectral curvature than can be accomodated by continuous injection models. Of the single burst models, as in Cygnus A, KP models usually fit somewhat better than the exponentiallysteepening JP models, although the signal-to-noise is rarely good enough to be definitive. In view of the fact that the JP steepening is an extreme case which will be flattened by any inhomogeneity in the model (c.f. section 2), this result is not threatening to the standard ageing scenario.

A more worrying trend, which also echos the results from Cyg A, is that in the hotspot the best-fit injection index is usually flatter than in the bridge, even in regions relatively close to the hotspot. For instance, in 3C 55, as shown by Fig. 3 , the Np hotspot is well fitted by a CI model with $\alpha_{0}=0.83 \pm 0.02$; in contrast, just one beamwidth $(30 \mathrm{kpc})$ down this very regular bridge, a $\mathrm{KP}$ model gives a $\chi^{2}$ of 0.9 compared to 6.9 for the CI model, and $\alpha_{0}=0.98 \pm 0.03$ (rather independently of the model). Furthermore, the break frequencies in the bridges close to the hotspots are often substantially higher than the $\nu_{T}$ derived by CI fits to the hotspots. These two results both imply that the "continuous flow" hotspot model of Meisenheimer et al. (1989) is much too simple, as discussed in detail by Carilli et al. (1991).

The "leaky hotspot" model of Blundell, Rawlings \& Willott (1999) is one attempt to deal with this problem. Here fluid exits the hotspot with a variety of histories, both of initial rapid ageing in the hotspot field, and subsequent adia- 


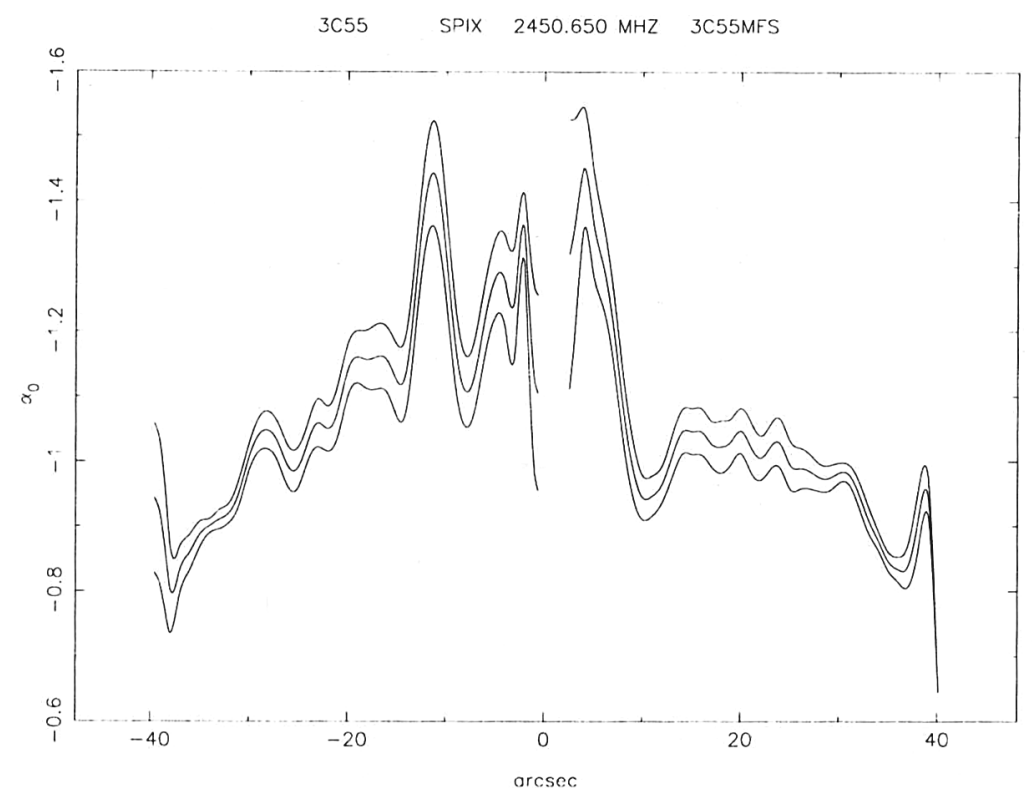

Figure 6. Profile of KP model injection index $\alpha_{0}$ along the bridge of 3C 55: the middle line gives the best-fit value and the outer two the $1 \sigma$ error range.

batic expansion, generating a spectrum that curves smoothly over a wide range of frequencies. It is important to realise that the basic features of conventional spectral ageing can be retained in this model: after some time has past, one still expects a break to a much steeper spectrum at about the same frequency as in the standard model, although the transition will be harder to pin-point because the low-frequency spectrum is already curved. Detailed spectral modelling is needed to see how clearly the break is retained.

An important question for spectral age determination is the constancy of $\alpha_{0}$ over the lobe. In the case of 3C 55 there is a clear tendency for $\alpha_{0}$ to increase towards the middle of the source, as $\nu_{T}$ falls. (Fig 6). This could again be a symptom of an incorrect spectral model, suggesting that the spectra curve more slowly than the KP model from which these parameters were derived.

\section{Inverse Compton Radiation}

Inverse-Compton radiation (ICR) in X-rays is a powerful diagnostic for the central unknowns in radio spectral ageing, because ICR depends on the relativistic electrons in just the same way as the synchrotron emission, substituting photon energy density for magnetic. For diffuse radio lobes, the CMB dominates the photon flux, so the equivalent field is (Mather et al. 1999):

$$
B_{\text {phot }}=(1+z) \times(0.3239 \pm 0.0002) \mathrm{nT}(!)
$$

The ICR directly gives the normalization of the electron energy spectrum, whose shape can be inferred from the radio emission (up to weighting factors caused by 
any variation in magnetic fields). In turn, this allows us to estimate a (weighted) $B$ from the synchrotron emission.

ICR was at the limit of detection with ROSAT and $A S C A$. The strongest sources are expected to be nearby giants since these combine high flux density with low estimated $B$, and therefore should be particularly rich in relativistic electrons. Tsakiris et al. (1996) observed 3 giant radio galaxies with ROSAT, detecting them all at the 3-7 $\sigma$ level after integrating over the region defined by the radio structure. The results imply $B \approx 0.5-1 B_{\text {me }}$ in all three cases. Prior to our work, For A was detected by Kaneda et al. (1995) and Feigelson et al. (1995), and afterwards Cen B by Tashiro (1998). Synchrotron self-Compton emission was detected in the hotspots of Cyg A and 3C 295 (Harris et al. 1994, 2000), and finally there is a claimed detection of ICR seeded by a hidden quasar in 3C 219 (Brunetti et al. 1999). In every one of these cases the inferred magnetic field is within a factor of two of the value derived by the conventional minimum energy calculation! The last paper is an apparent exception only because the authors made a self-consistent equipartition calculation, instead of blindly applying the standard formula (Brunetti, priv. comm.).

Thus, whether or not equipartition actually takes place, the usual formula (e.g. Miley 1980) seems to give relatively good values for $B$. There is evidence that this is really a coincidence (Leahy \& Gizani 2000), and there are particle energy components omitted from the conventional analysis that actually dominate the total energy; but in the present context we are only interested in $B$ and the relativistic electron normalization, and for these it seems that we have been right all along. A caveat is that at present ICR can only be detected if $B \lesssim B_{\text {me }}$, so there is a partial selection effect; however it is certainly true from many upper limits that it is at best rare for $B \ll B_{\text {me }}$. New X-ray observations with Chandra and $X M M$ will soon greatly clarify this area.

\section{Conclusions}

Spectral ageing has to be important in radio galaxies, and the data are consistent with its qualitative predictions. Inverse-Compton results suggest that our magnetic field estimates are in pretty good shape. Complications over the standard picture mean that ages are not precise and are likely to be underestimates, but uncertainties should be less than a factor of three.

Our principle worry is now the discrepancy between hotspot and lobe injection indices, and the possible variation of $\alpha_{0}$ within the lobes. The former may indicate that hotspots are "leaky" as suggested by Blundell et al. (1999), or more radically that diffuse particle acceleration occur in the lobes as well as the hotspots. These possibilities are difficult to put to a clear test, although any systematic patterns in the $\alpha_{0}$ distribution within lobes may favour one or the other.

We have also found two hotspots with $\alpha_{0}<0.5$, suggesting Fermi acceleration at relativistic shocks, consistent with current ideas about jet speeds.

Acknowledgments. We thank Robert Laing and Peter Stephens for their work in the early stages of this project, and Steve Spangler for some images of $3 \mathrm{C} 79$. 


\section{References}

Alexander P., 1987, MNRAS, 225, 27

Baars J. W. M., Genzel R., Pauliny-Toth I. I. K., Witzel A., 1977, A\&A, 61, 99

Blundell K. M., Rawlings S., Willott C. J., 1999, AJ, 117, 677

Brunetti G., Comastri A., Setti G., Feretti L., 1999, A\&A, 342, 57

Burch S. F., 1977, MNRAS, 180, 623

Carilli C. L., Perley R. A., Dreher J. W., Leahy J. P., 1991, ApJ, 383, 554

Dennett-Thorpe J., Bridle A. H., Scheuer P. A. G., Laing R. A., Leahy J. P., 1997, MNRAS, 289, 753

Feigelson E. D., Laurent-Muehleisen S. A., Kollgaard R. I., Fomalont E. B., 1995, ApJ, 449, L149

Harris D. E., Carilli C. L., Perley R. A., 1994, Nat, 367, 713

Harris D. E. et al., 2000, ApJ, 530, L81

Heavens A., 1989, in Meisenheimer K., Röser H.-J., eds, Hot Spots in Extragalactic Radio Sources, Springer, Berlin p. 247

Kaneda H. et al., 1995, ApJ, 453, L13

Kardashev N. S., 1962, Soviet Astr., 6, 317

Katz-Stone D. M., Rudnick L., Anderson M. C., 1993, ApJ, 407, 549

Kirk J. G., Heavens A. F., 1989, MNRAS, 239, 995

Laing R. A., Peacock J. A., 1980, MNRAS \& Microfiche MN, 190, 903

Laing R. A., Riley J. M., Longair M. S., 1983, MNRAS, 204, 151

Leahy J. P., Gizani N. A. B., 2000, New Astr.Rev., in press

Leahy J. P., Muxlow T. W. B., Stephens P. W., 1989, MNRAS, 239, 401

Leahy J. P., 1991, in Hughes P. A., ed, Beams and Jets in Astrophysics, CUP, Cambridge, p. 100

Mather J. C., Fixsen D. J., Shafer R. A., Mosier C., Wilkinson D. T., 1999, ApJ, 512, 511

Meisenheimer K., Röser H.-J., Hiltner P. R., Yates M. G., Longair M. S., Chini R., Perley R. A., 1989, A\&A, 219, 63

Miley, G., 1980, ARA\&A, 165

Rudnick L., 2000, New Astr.Rev, in press

Tashiro M. et al., 1998, ApJ, 499, 713

Tribble P. C., 1993, MNRAS, 261, 57

Tsakiris D., Leahy J. P., Strom R. G., Barber C. R., 1996, in Ekers R. D., Fanti C., Padrielli L., eds, Extragalactic Radio Sources, I.A.U. Symp. 175, Kluwer, Dordrecht, p. 256 